\title{
2135. Large amplitude free vibrations of simply supported moderately thick rectangular plates using coupled displacement field method
}

\author{
Krishna Bhaskar $\mathbf{K}^{\mathbf{1}}$, Meera Saheb $\mathrm{K}^{\mathbf{2}}$ \\ University College of Engineering (A), JNTUK Kakinada, Andhra Pradesh, India \\ ${ }^{1}$ Corresponding author \\ E-mail: ${ }^{1}$ krisbhaskar22@yahoo.co.in, ${ }^{2}$ meera.aec@gmail.com
}

Received 1 February 2016; received in revised form 24 May 2016; accepted 28 June 2016

DOI http://dx.doi.org/10.21595/jve.2016.16889

\begin{abstract}
In this paper a novel method known as Coupled Displacement Field Method was proposed to evaluate the large amplitude free vibration behavior of the moderately thick rectangular plates with simply supported boundary conditions. Here a single term trigonometric admissible displacement field was assumed for one of the variables, say the total rotations (in both $X, Y$ directions). With the help of the coupling equations, the spatial variation for the lateral displacement field is derived in terms of the total rotations, where the two independent variables problem becomes one. The coupled displacement field method makes use of the energy formulation which contains half the number of unknown independent coefficients, in the case of a rectangular plate, when compared to the conventional Rayleigh-Ritz method. Closed form expressions for the linear and nonlinear fundamental frequency parameters for the all edges simply supported moderately thick rectangular plates are derived. The numerical results obtained from the present formulation are validated with those obtained from the existing literature for the given moderately thick plates.
\end{abstract}

Keywords: coupled displacement field method, large amplitude vibrations, moderately thick rectangular plates.

\section{Introduction}

Evaluation of free vibration behavior of a structure is an essential consideration in the design of a structure. Understanding the fundamental frequency parameters for moderately thick rectangular plates will be advantageous in the design of structural members at the initial design phase. The conventional energy methods provide an effective means in evaluating the fundamental frequency parameters of these structural members and the results obtained by these approaches will act as an upper limit for comparison.

The concept of coupled displacement field (CDF) which was successfully used in the finite element (FE) analysis and reported in the open literature. However much attention was not received in the continuum correlation of the Finite element analysis combined with the CDF in the past years except in the formulation presented by Zhou [1] where the two independent variables (transverse displacement and rotation) are integrated using coupling equation. The large amplitude free vibration behavior of uniform Timoshenko beams using CDF method was successfully demonstrated [2]. In this study, using CDF method the large amplitude free vibration behavior of uniform shear deformable moderately thick rectangular plates was studied and exact closed form solutions were obtained. For the thin plates the fundamental frequency parameter was evaluated in [3-10] for several configurations and boundary conditions. Nonlinear analysis of plates was evaluated by using finite element method $[4,13]$. S. H. Hashemi et al. [11] used dimensionless equation of motion and solved the problem based on theory to study the transverse vibration behavior of thick rectangular plates. In Rayleigh-Ritz method we need to assume an $\mathrm{n}$ term admissible trial function for the total rotation and another $\mathrm{n}$ term admissible trial function for the transverse displacement for solving for solving free vibration analysis of Mindlin plate. This result in the vibration problems to have $2 n$ unknown undetermined coefficients, in general and the use of the classical Rayleigh Ritz method $[12,14]$ contain $2 \mathrm{n}$ homogeneous simultaneous 
equations which are to be solved in order to obtain fundamental frequency parameter.

In this paper the independent fields for total rotation and the transverse displacement are coupled by using coupling equations, which are derived from static equilibrium equations in order to obtain static solution for shear deformable moderately thick rectangular plates. This methodology reduces the magnitude of the problem by reducing the number of unknown coefficients from $2 \mathrm{n}$ to $\mathrm{n}$ when compared with classical Rayleigh Ritz method. The practicality of CDF method is verified by solving the vibration problem considering uniform and isotropic moderately thick rectangular plates with all edges simply supported boundary conditions Fig. 1. The numerical results obtained by this method are compared with the results obtained from the finite element method [12] and other researchers. The comparison quantifies the effectiveness of the proposed CDF method.



Fig. 1. Uniform all edges simply supported moderately thick rectangular plates

\section{Coupled displacement field (CDF) method for plates}

In this section the detailed procedure for evaluating fundamental linear frequency parameter of uniform all edges simply supported moderately thick rectangular plate based on CDF method is discussed. Here an admissible trial functions for $\theta_{x}$ and $\theta_{y}$ are assumed in the functional form which satisfy the boundary conditions and symmetric conditions for the fundamental mode as:

$\theta_{x}=\alpha \frac{\pi}{a} \cos \frac{\pi x}{a} \sin \frac{\pi y}{b}$

$\theta_{y}=\alpha \frac{\pi}{b} \sin \frac{\pi x}{a} \cos \frac{\pi y}{b}$.

The coupling equations for evaluating the transverse displacement distribution $w$ in $x$ and $y$ directions are given as:

$$
\begin{aligned}
& \frac{d w}{d x}=-\theta_{x}+\frac{h^{2}}{3.5}\left[\frac{\partial^{2} \theta_{x}}{\partial x^{2}}+v \frac{\partial^{2} \theta_{y}}{\partial y \partial x}\right]+\frac{h^{2}}{10}\left[\frac{\partial^{2} \theta_{x}}{\partial y^{2}}+\frac{\partial^{2} \theta_{y}}{\partial y \partial x}\right], \\
& \frac{d w}{d y}=-\theta_{y}+\frac{h^{2}}{3.5}\left[\frac{\partial^{2} \theta_{y}}{\partial y^{2}}+v \frac{\partial^{2} \theta_{x}}{\partial y \partial x}\right]+\frac{h^{2}}{10}\left[\frac{\partial^{2} \theta_{y}}{\partial x^{2}}+\frac{\partial^{2} \theta_{x}}{\partial y \partial x}\right],
\end{aligned}
$$

substituting Eq. (1) and Eq. (2) in Eq. (3) and Eq. (4) we get:

$$
\begin{aligned}
& \frac{d w}{d x}=-\alpha p \cos p x \sin q y\left[1+\frac{h^{2}}{3.5}\left(p^{2}+v q^{2}\right)+\frac{h^{2}}{5}\left(q^{2}\right)\right], \\
& \frac{d w}{d y}=-\alpha q \sin p x \cos q y\left[1+\frac{h^{2}}{3.5}\left(q^{2}+v p^{2}\right)+\frac{h^{2}}{5}\left(p^{2}\right)\right] .
\end{aligned}
$$

Integrating above equations and after evaluating the constant of integration as: 
$w_{x}=-\alpha \sin p x \sin q y\left[1+\frac{h^{2}}{3.5}\left(p^{2}+v q^{2}\right)+\frac{h^{2}}{5}\left(q^{2}\right)\right]$,
$w_{y}=-\alpha \sin p x \sin q y\left[1+\frac{h^{2}}{3.5}\left(q^{2}+v p^{2}\right)+\frac{h^{2}}{5}\left(p^{2}\right)\right]$,

where $p=\pi / a$ and $q=\pi / b$. The expression for strain energy of moderately thick rectangular plate is given as:

$$
\begin{aligned}
U & =\frac{D}{2} \int_{0}^{b} \int_{0}^{a}\left\{\left(\frac{\partial \theta_{x}}{\partial x}\right)^{2}+\left(\frac{\partial \theta_{y}}{\partial y}\right)^{2}+2 v \frac{\partial \theta_{x}}{\partial x} \frac{\partial \theta_{y}}{\partial y}+2(1-v) \frac{\partial \theta_{x}}{\partial y} \frac{\partial \theta_{y}}{\partial x}\right\} d x d y \\
& +\frac{k G h}{2} \int_{0}^{b} \int_{0}^{a}\left\{\left(\frac{d w}{d x}+\theta_{x}\right)^{2}+\left(\frac{d w}{d y}+\theta_{y}\right)^{2}\right\} d x d y .
\end{aligned}
$$

Substituting Eq. (1, 2), Eq. (5, 6) in Eq. (9) and after simplification we get strain energy as:

$$
U=\frac{D}{2} \alpha^{2} a b\left\{\left(p^{2}+q^{2}\right)^{2}+\left(\frac{k 6(1-v)}{h^{2}}\left(p^{2}(A-1)^{2}+q^{2}(B-1)^{2}\right)\right)\right\},
$$

where:

$$
A=\left[1+\frac{h^{2}}{3.5}\left(p^{2}+v q^{2}\right)+\frac{h^{2}}{5}\left(q^{2}\right)\right], \quad B=\left[1+\frac{h^{2}}{3.5}\left(q^{2}+v p^{2}\right)+\frac{h^{2}}{5}\left(p^{2}\right)\right]
$$

The expression for kinetic energy of moderately thick rectangular plate plate is given as:

$$
T=\frac{\rho h w_{L}^{2}}{2} \int_{0}^{b} \int_{0}^{a}\left[w^{2}+\frac{h^{2}}{12}\left(\theta_{x}^{2}+\theta_{y}^{2}\right)\right] d x d y
$$

Substituting Eq. $(1,2)$ and Eq. (7) in Eq. (11) and after simplification we get:

$$
T=\frac{\rho h \omega_{L}^{2}}{2} \alpha^{2} \frac{a b}{4}\left(A^{2}+\frac{h^{2}}{12}\left(p^{2}+q^{2}\right)\right) \text {. }
$$

By minimizing the Lagranzian with respect to undetermined coefficient $\alpha$ we get the fundamental frequency parameter $\lambda$ :

$$
\frac{\partial(U-T)}{\partial \alpha}=0 .
$$

\section{Large amplitude vibrations}

The principle of conservation of total energy states that at any instant of time total energy is constant for any vibrating structure neglecting damping.

From the derived transverse displacement field $w$ for an assumed $\theta_{x}$ and $\theta_{y}$ the strain energy and the kinetic energy in the plate are derived and is discussed in the previous section. In order to evaluate the large amplitude free vibrations of the plate the work done due to the stretching of the central plane of the plate has to be derived. The expression for tension developed in the plate due 
to large amplitudes in $x$ direction is given as:

$T_{x}=\frac{E h}{2 a} \int_{0}^{a}\left\{\frac{d w(x)}{d x}\right\}^{2} d x$,

$T_{x}=\frac{E h}{4} \alpha^{2} \frac{\pi^{2}}{a^{2}}$

Shear flexible terms are not involved in the expression of tension because the tension in the plate is only caused by stretching of the mid plane due to bending and not due to shear. Similarly, the tension in y direction is given as:

$T_{y}=\frac{E h}{2 b} \int_{0}^{b}\left\{\frac{d w(y)}{d y}\right\}^{2} d y$,

$T_{y}=\frac{E h}{4} \alpha^{2} \frac{\pi^{2}}{b^{2}}$.

The expression for work done is given as:

$W=\frac{1}{2} \int_{0}^{b} \int_{0}^{a}\left\{\frac{1}{2} T_{x}\left(\frac{d w}{d x}\right)^{2}+\frac{1}{2} T_{y}\left(\frac{d w}{d y}\right)^{2}\right\} d x d y$.

Substituting Eq. (5-6), Eq. (17) and Eq. (19) in Eq. (18) and after simplification we get:

$W=\alpha^{4} \frac{E h}{64} a^{2} x\left(p^{4} A^{2}+q^{4} B^{2}\right)$.

By principle of conservation of total energy:

$U+T+W=$ constant.

Substituting Eq. (10), Eq. (12) and Eq. (19) in Eq. (20) and after simplification:

$\dot{z}^{2}+\alpha_{1} z^{2}+\alpha_{2} z^{4}=$ constant.

Substituting $D=E h^{3} / 12\left(1-v^{2}\right), b=a x$ and after simplification

$\lambda=\alpha_{1}=\frac{\rho h \omega_{L}^{2} a^{4}}{D}=\frac{\pi^{4}\left[\left(1+\left(\frac{1}{x}\right)^{2}\right)^{2}+k 6(1-v) \pi^{2}\left(A_{1}^{2}+\left(\frac{B_{1}}{x}\right)^{2}\right)\left(\frac{h}{a}\right)^{2}\right]}{\left[\left(1+\pi^{2} A_{1}\left(\frac{h}{a}\right)^{2}\right)^{2}+\frac{\pi^{2}}{12}\left(1+\left(\frac{1}{x}\right)^{2}\right)\left(\frac{h}{a}\right)^{2}\right]}$,
$\alpha_{2}=\frac{\rho h \omega_{N L}^{2} a^{4}}{D}=\frac{\pi^{4} \frac{3}{2}\left(1-v^{2}\right)\left[\left(1+A_{1} \pi^{2}\left(\frac{h}{a}\right)^{2}\right)^{2}+\frac{1}{x^{4}}\left(1+B_{1} \frac{\pi^{2}}{x^{2}}\left(\frac{h}{a}\right)^{2}\right)^{2}\right]}{\left[\left(1+\pi^{2} A_{1}\left(\frac{h}{a}\right)^{2}\right)^{2}+\frac{\pi^{2}}{12}\left(1+\left(\frac{1}{x}\right)^{2}\right)\left(\frac{h}{a}\right)^{2}\right]\left(\frac{\alpha_{m}}{h}\right)^{-2}}$,

where: 
$A_{1}=\frac{1}{3.5}\left(1+v \frac{1}{x^{2}}\right)+\frac{1}{5 x^{2}}, \quad B_{1}=\frac{1}{3.5}\left(\frac{1}{x^{2}}+v\right)+\frac{1}{5}, \quad b=a x$.

By applying harmonic balance method [2] and simplifying, the expression for frequency ratio can be written as:

$\left[\frac{\omega_{N L}}{\omega_{L}}\right]^{2}=1+\frac{9}{4}\left(\frac{\left(1-v^{2}\right)\left[\left(1+A_{1} \pi^{2}\left(\frac{h}{a}\right)^{2}\right)^{2}+\frac{1}{x^{4}}\left(1+B_{1} \frac{\pi^{2}}{x^{2}}\left(\frac{h}{a}\right)^{2}\right)^{2}\right]\left(\frac{\alpha_{m}}{h}\right)^{2}}{\left[\left(1+\left(\frac{1}{x}\right)^{2}\right)^{2}+k 6(1-v) \pi^{2}\left(A_{1}^{2}+\left(\frac{B_{1}}{x}\right)^{2}\right)\left(\frac{h}{a}\right)^{2}\right]}\right)$.

The Eq. (24) gives the expression for frequency ratio in terms of plate thickness ratio $(h / a)$ and maximum amplitude ratio $\left(\alpha_{m} / h\right)$.

Table 1. Values of linear fundamental frequency parameter $(\sqrt{\lambda})$ for all edges simply supported moderately thick rectangular plate at different aspect ratios.

\begin{tabular}{|c|c|c|c|c|c|c|c|c|}
\hline \multicolumn{8}{|c|}{ Aspect ratio $(b / a)$} \\
\hline$\frac{h}{a}$ & \multicolumn{2}{|c|}{1} & \multicolumn{2}{c|}{1.5} & \multicolumn{2}{c|}{2} & \multicolumn{2}{c|}{2.5} \\
\cline { 2 - 9 } & CDF & Ref & CDF & Ref & CDF & Ref & CDF & Ref \\
\hline 0.001 & 19.7391 & $19.7415^{*}$ & 14.2560 & $14.2561 \mathrm{a}$ & 12.3370 & $12.3389^{*}$ & 11.4487 & $11.4487 \mathrm{a}$ \\
& & $19.7362 \mathrm{a}$ & & $14.2566 \mathrm{~d}$ & & $12.3370 \mathrm{~d}$ & & $11.4507 \mathrm{~d}$ \\
\hline 0.05 & 19.5152 & $19.7395 \mathrm{~d}$ & & & & & & \\
\hline 0.1 & $18.2271^{*}$ & 14.1095 & $14.1662 \mathrm{c}$ & 12.2224 & $12.1770^{*}$ & 11.3485 & $11.3906 \mathrm{c}$ \\
& & $19.5676 \mathrm{c}$ & & & & $12.2696 \mathrm{c}$ & & \\
\hline & & $18.3464^{*}$ & 13.6917 & $13.898 \mathrm{~b}$ & 11.8935 & $11.8515^{*}$ & 11.0598 & $11.2158 \mathrm{~b}$ \\
& & $19.0650 \mathrm{~b}$ & & $13.9085 \mathrm{c}$ & & $12.0675 \mathrm{~b}$ & & $11.226 \mathrm{c}$ \\
\hline 0.15 & $17.0840 \mathrm{c}$ & & $13.8984 \mathrm{~d}$ & & $12.0752 \mathrm{c}$ & & $11.2158 \mathrm{~d}$ \\
& & $19.0651 \mathrm{~d}$ & & & & $12.0676 \mathrm{~d}$ & & \\
\hline 0.2 & 16.8857 & $17.3727 *$ & 13.0600 & $13.5147 \mathrm{c}$ & 11.3900 & $11.4468^{*}$ & 10.6154 & $10.9617 \mathrm{c}$ \\
& & $18.3661 \mathrm{c}$ & & & & $11.7747 \mathrm{c}$ & & \\
\hline
\end{tabular}

\section{Numerical results and discussion}

Using the formulation described above, the large amplitude vibration behavior of a uniform moderately thick rectangular plates for different aspect ratios were obtained in terms of $\omega_{N L} / \omega_{L}$ or $T_{N L} / T_{L}$ (Ratio of non-linear radian frequency to the linear radian frequency or non-linear time period to the linear time period) in terms of various $\alpha_{m} / h$ (maximum amplitude ratios) and $\mathrm{h} / \mathrm{a}$ (plate thickness ratios). As a demonstration of the proposed formulation, the moderately thick rectangular plates are considered with axially immovable edges (Fig. 1).

The present results in terms of fundamental frequency parameter are presented in Table 1 for all edges simply supported moderately thick rectangular plate. For comparison and validation of the proposed method, similar results obtained by the finite element method [4] and other researchers are included in Table 1. From Table 1 it is found that for a particular given aspect ratio the fundamental frequency parameter decreases with increase of plate thickness ratio. For a given aspect ratio of 2.5 , further reduction in frequency parameter is observed in case of moderately thick plates.

The present results in terms of $T_{N L} / T_{L}$ which is the reciprocal of $\omega_{N L} / \omega_{L}$ for all edges simply 
supported moderately thick rectangular plate are given in Table 2 to Table 5 respectively for different aspect ratios. The present results for the extreme case of $\alpha_{m} / h=1$ and $h / a=0.2$ match very well with the results of FEM and other researchers. It is found from the Table 2 to Table 4 that the frequencies are increasing with increase of amplitude ratio. More nonlinearity is observed with the increase in aspect ratio in rectangular plates when compared to square plates.

Further, the present results match excellently with those of [13] for the thin plates, showing the efficacy of the proposed method and the accuracy of the results over a range of $h / a=0.001$ and $h / a=0.2$. Further, no shear locking phenomenon exists in the present formulation.

Table 2. $T_{N L} / T_{L}$ values of all edges simply supported moderately thick square plate $(b / a=1)$

\begin{tabular}{|c|c|c|c|c|c|c|c|c|c|c|c|c|}
\hline \multirow{3}{*}{$\frac{h}{a}$} & \multicolumn{12}{|c|}{$\alpha_{m} / h$} \\
\hline & \multicolumn{2}{|r|}{0} & \multicolumn{2}{|r|}{0.2} & \multicolumn{2}{|r|}{0.4} & \multicolumn{2}{|r|}{0.6} & \multicolumn{2}{|r|}{0.8} & \multicolumn{2}{|r|}{1.0} \\
\hline & CDF & FEM [4] & $\mathrm{CDF}$ & FEM [4] & $\mathrm{CDF}$ & FEM [4] & $\mathrm{CDF}$ & FEM [4] & $\mathrm{CDF}$ & FEM [4] & $\mathrm{CDF}$ & FEM [4] \\
\hline 0.001 & 1 & 1 & 0.9801 & $\begin{array}{c}0.9818 \\
0.9821 * \\
0.9809 \$ \\
0.9783 \#\end{array}$ & 0.9269 & $\begin{array}{c}0.9327 \\
0.9338 * \\
0.9297 \$ \\
0.9210 \#\end{array}$ & 0.8548 & $\begin{array}{c}0.8652 \\
0.8673^{*} \\
0.8602 \$ \\
0.8451 \#\end{array}$ & 0.7773 & $\begin{array}{c}0.7916 \\
0.7943^{*} \\
0.7853 \$ \\
0.7653\end{array}$ & 0.7029 & $\begin{array}{c}0.7199 \\
0.7233 * \\
0.7131 \$ \\
0.6901 \#\end{array}$ \\
\hline 0.05 & 1 & 1 & 0.9798 & 0.9807 & 0.9257 & 0.9290 & 0.8526 & 0.8586 & 0.7744 & 0.7827 & 0.6996 & 0.7098 \\
\hline 0.1 & 1 & 1 & 0.9787 & 0.9788 & 0.9220 & 0.9226 & 0.8462 & 0.8475 & 0.7658 & 0.7681 & 0.6898 & 0.6931 \\
\hline 0.15 & 1 & 1 & 0.9768 & 0.9766 & 0.9159 & 0.9153 & 0.8356 & 0.8350 & 0.7519 & 0.7520 & 0.6741 & 0.6752 \\
\hline 0.2 & 1 & 1 & 0.9742 & 0.9740 & 0.9074 & \begin{tabular}{|l|l|}
+0.9069 \\
\end{tabular} & 0.8213 & 0.8212 & 0.7337 & 0.7347 & 0.6537 & 0.6562 \\
\hline
\end{tabular}

Table 3. $T_{N L} / T_{L}$ values of all edges simply supported moderately thick plate $(b / a=1.5)$

\begin{tabular}{|c|c|c|c|c|c|c|}
\hline \multirow{2}{*}{$\frac{7}{a}$} & \multicolumn{6}{|c|}{$\alpha_{m} / h$} \\
\cline { 2 - 7 } & 0 & 0.2 & 0.4 & 0.6 & 0.8 & 1 \\
\hline 0.001 & 1 & 0.9773 & 0.9174 & 0.8383 & 0.7555 & 0.6780 \\
\hline 0.05 & 1 & 0.9769 & 0.9163 & 0.8363 & 0.7529 & 0.6751 \\
\hline 0.1 & 1 & 0.9759 & 0.9128 & 0.8303 & 0.7452 & 0.6665 \\
\hline 0.15 & 1 & 0.9741 & 0.9069 & 0.8204 & 0.7326 & 0.6525 \\
\hline 0.2 & 1 & 0.9715 & 0.8986 & 0.8067 & 0.7154 & 0.6337 \\
\hline
\end{tabular}

Table 4. $T_{N L} / T_{L}$ values of all edges simply supported moderately thick rectangular plate $(b / a=2)$

\begin{tabular}{|c|c|c|c|c|c|c|c|c|c|c|c|c|}
\hline \multirow{3}{*}{$\frac{h}{a}$} & \multicolumn{12}{|c|}{$\alpha_{m} / h$} \\
\hline & \multicolumn{2}{|c|}{0} & \multicolumn{2}{|c|}{0.2} & \multicolumn{2}{|c|}{0.4} & \multicolumn{2}{|c|}{0.6} & \multicolumn{2}{|c|}{0.8} & \multicolumn{2}{|c|}{1.0} \\
\hline & $\mathrm{CDF}$ & $\begin{array}{c}\text { FEM } \\
{[4]}\end{array}$ & $\mathrm{CDF}$ & $\begin{array}{c}\text { FEM } \\
{[4]}\end{array}$ & CDF & $\begin{array}{c}\text { FEM } \\
{[4]}\end{array}$ & $\mathrm{CDF}$ & $\begin{array}{c}\text { FEM } \\
\text { [4] }\end{array}$ & $\mathrm{CDF}$ & $\begin{array}{c}\text { FEM } \\
{[4]}\end{array}$ & $\mathrm{CDF}$ & $\begin{array}{c}\text { FEM } \\
{[4]}\end{array}$ \\
\hline 0.001 & 1 & 1 & 0.9733 & 0.9768 & 0.9043 & 0.9161 & 0.8162 & 0.8367 & 0.7272 & 0.7550 & 0.6465 & 0.6795 \\
\hline 0.05 & 1 & 1 & 0.9729 & 0.9761 & 0.9031 & 0.914 & 0.8141 & 0.8332 & 0.7245 & 0.7506 & 0.6436 & 0.6747 \\
\hline 0.1 & 1 & 1 & 9717 & 0.9749 & 0.8992 & 0.91 & 0.8076 & 0.8266 & 0.717 & 0.7423 & 0.6351 & 0.6658 \\
\hline 0.15 & 1 & 1 & 0. & 0.9733 & 0.8928 & 0.9049 & 0.7973 & 0.8186 & 0.7038 & 0.7325 & 0.6212 & 0.6554 \\
\hline 0.2 & 1 & 1 & 0.9667 & 0.9714 & 0.8836 & 0.899 & 0.7829 & 0.8092 & 0.6864 & 0.7213 & 0.6025 & 0.6437 \\
\hline
\end{tabular}

Table 5. $T_{N L} / T_{L}$ values of all edges simply supported moderately thick rectangular plate $(b / a=2.5)$

\begin{tabular}{|c|c|c|c|c|c|c|}
\hline \multirow{2}{*}{$\frac{\mid}{a}$} & \multicolumn{6}{|c|}{$\alpha_{m} / h$} \\
\cline { 2 - 7 } & 0 & 0.2 & 0.4 & 0.6 & 0.8 & 1 \\
\hline 0.001 & 1 & 0.9702 & 0.8945 & 0.8002 & 0.7073 & 0.6249 \\
\hline 0.05 & 1 & 0.9697 & 0.8932 & 0.7980 & 0.7047 & 0.6221 \\
\hline 0.1 & 1 & 0.9684 & 0.8892 & 0.7914 & 0.6968 & 0.6137 \\
\hline 0.15 & 1 & 0.9663 & 0.8824 & 0.7809 & 0.6840 & 0.6001 \\
\hline 0.2 & 1 & 0.9631 & 0.8728 & 0.7662 & 0.6666 & 0.5818 \\
\hline
\end{tabular}

\section{Conclusions}

The complex structures in various fields of engineering such as Aerospace, Mechanical, Civil, 
Naval, Nuclear, and Automobile are made up of simple structural members like beams, plates (thin or moderately thick) and shells. Due to functional requirements sometimes these structural members may be either thin or moderately thick. If these structural members are subjected to severe dynamic environment, they vibrate with large amplitudes. The authors evaluated free vibration response i.e. natural frequencies both in the linear and nonlinear regime.

The concept of coupled displacement field method (CDF) is successfully applied to study the large amplitude free vibrations of all edges simply supported moderately thick rectangular plate for different aspect ratios. Elegant and closed form expressions are derived in terms of maximum amplitude ratio $\left(\alpha_{m} / h\right)$ and plate thickness ratio $h / a$ using single term admissible functions for the total rotations $\theta_{x}$ and $\theta_{y}$. The closed form expressions are obtained for non-dimensional frequency parameter as a function of thickness ratio $h / a$ and frequency ratio or the time period ratio as a function of maximum amplitude ratio $\alpha_{m} / h$ and plate thickness ratio $h / a$. Comparison of present results wherever possible for the first mode of vibration with the available open literature shows that the coupled displacement field method gives accurate values for the linear frequency parameter and ratios of nonlinear to linear frequencies.

The natural frequencies are useful for the design engineers for the purpose of design of various structural members. Using the results given in Tables 1-6 failure of the structural member can be predicted based on the stiffness approach.

\section{References}

[1] Zhou D. Free vibration of multi-span Timoshenko beams using static Timoshenko beam functions. Journal of Sound and Vibration, Vol. 241, 2001, p. 725-734.

[2] Rao G. V., MeeraSaheb K., Rangajanardhana G. Concept of coupled displacement field for large amplitude free vibrations of shear flexible beams. American Society of Mechanical Engineering, Vol. 128, 2006, p. 251-255.

[3] Mindlin R. D. Influence of rotary inertia and shear on flexural motions of isotropic elastic plates. Journal of Applied Mechanics, Vol. 18, 1951, p. 31-38.

[4] Raju K. K., Rao G. V., Raju I. S. Effect of geometric nonlinearity on large amplitude free flexural vibrations of moderately thick rectangular plates. Computers and Structures, Vol. 9, 1978, p. 441-444.

[5] Wah T. Large amplitude flexural vibration of rectangular plates. International Journal of Mechanical Sciences, Vol. 5, 1963, p. 425-438.

[6] Chu H. N., Herrman G. Influence of large amplitudes on free flexural vibrations of rectangular elastic plates. Journal of Applied Mechanics, Vol. 23, 1956, p. 532-540.

[7] Chu Mei Finite element displacement method for large amplitude free flexural vibrations of beams and plates. Computers and Structures, Vol. 3, 1973, p. 163-174.

[8] Wang C. M., Wang C. Y., Reddy J. N. Exact Solutions for Buckling of Structural Members. CRC Press, 2005.

[9] Leissa W. The free vibration of rectangular plates. Journal of Sound and Vibration, Vol. 31, Issue 3, 1973, p. 257-293.

[10] Milan Batista Analytical solution for free vibrations of simply supported transversally inextensible homogeneous rectangular plate. arXiv:1007.2539, 2010.

[11] Hashemi H. S., Arsanjani M. Exact characteristic equations for some of classical boundary conditions of vibrating moderately thick rectangular plates. International Journal of Solids and Structures, Vol. 42, 2005, p. 819-853.

[12] Liew K. M., Xiang Y., Kitipornchai S. Transverse vibration of thick rectangular plates. Part 1: Comprehensive sets of boundary conditions. Computers and Structures, Vol. 49, 1993, p. 1-29.

[13] Raju K. K., Hinton E. Natural frequencies and modes of rhombic Mindlin plates. Earthquake Engineering Structural Dynamics, Vol. 8, 1980, p. 55-62.

[14] Gorman D. J. Free Vibration Analysis of Rectangular Plates. Elsevier North Holland, New York. 


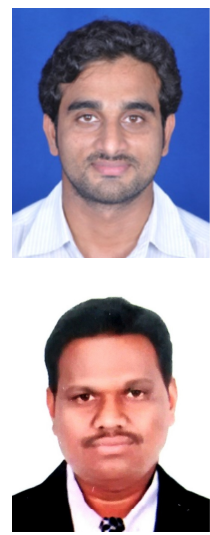

Krishna Bhaskar $\mathbf{K}$ is a Ph.D. research scholar working in the field of nonlinear vibrations. He is working as Assistant Professor in the Department of Mechanical Engineering, University College of Engineering, JNT University Kakinada, Andhra Pradesh India.

Meera Saheb K received Ph.D. degree in nonlinear vibrations, from Department of Mechanical Engineering, JNT University, Hyderabad, India in the year 2010. Now he is working as an Associate Professor in the Department of Mechanical Engineering, University College of Engineering, JNT University Kakinada, Andhra Pradesh, India. His current research interests are nonlinear vibrations, buckling, post buckling and nano composites. 